\title{
Respiración de Cheyne-Stokes en una adolescente con insuficiencia cardíaca secundaria a miocardiopatía dilatada que requirió trasplante cardíaco Cheyne-Stokes respiration in an adolescent with heart failure secondary to dilated cardiomyopathy requiring heart transplantation
}

Dra. Carla Pereyra ${ }^{a}$, Dr. Javier Fraire ${ }^{a}$, Dr. Lorenzo Olivero ${ }^{a}$ y Dr. Augusto Pérez ${ }^{b}$

\section{RESUMEN}

La respiración de Cheyne-Stokes es una variante cíclica o tipo de apnea central del sueño poco frecuente en la población pediátrica. Se describe a una paciente de 12 años con insuficiencia cardíaca grave relacionada con miocardiopatía dilatada que demostró trastornos del sueño con características de respiración de Cheyne-Stokes, que se resolvieron completamente después del trasplante cardíaco.

Palabras clave: respiración de Cheyne-Stokes, pediatría, sindromes de la apnea del sueño, trasplante de corazón.

\section{ABSTRACT}

Cheyne-Stokes respiration is a cyclic variant or type of central sleep apnea rare in pediatric population. We describe a 12-yearold female patient with severe heart failure related to dilated cardiomyopathy who demonstrated sleep disorders with Cheyne-Stokes breathing features, which completely resolved following cardiac transplantation.

Key words: Cheyne-Stokes respiration, pediatrics, sleep apnea syndromes, heart transplantation.

http: / / dx.doi.org/10.5546/aap.2021.e75

Cómo citar: Pereyra C, Fraire J, Olivero L, Pérez A. Respiración de Cheyne-Stokes en una adolescente con insuficiencia cardíaca secundaria a miocardiopatía dilatada que requirió trasplante cardíaco. Arch Argent Pediatr 2021;119(1):e75-e79.

a. Servicio de Clínica Pediátrica, Sección de Trastornos Respiratorios del Sueño Pediátrico.

b. Unidad de Cuidados Intensivos Pediátricos.

Hospital Italiano de Buenos Aires, Ciudad Autónoma de Buenos Aires, Argentina.

Correspondencia:

Dra. Carla Pereyra: carla.pereyra@hospitalitaliano.org.ar

Financiamiento: Ninguno.

Conflicto de intereses: Ninguno que declarar.

Recibido: 14-7-2020

Aceptado: 31-8-2020

\section{INTRODUCCIÓN}

La respiración de Cheyne-Stokes (RCS) es una variante cíclica del síndrome de apneas centrales poco frecuente en la población pediátrica. Se caracteriza por un patrón de respiración periódica, creciente-decreciente, que alterna con apneas y/o hipopneas centrales.

Representa un trastorno prevalente en adultos con insuficiencia cardíaca $(\text { el } 37 \%)^{1,2}$ y es predictor de mortalidad. ${ }^{3}$ En pediatría, la miocardiopatía dilatada (MCD) es una causa poco frecuente de insuficiencia cardíaca, con alta tasa de mortalidad e indicación de trasplante cardíaco en esta población. Hasta la actualidad, en edades pediátricas, solo ha sido publicado un caso de MCD y RCS que recibió trasplante cardíaco, con resolución del trastorno a tres meses de la intervención. ${ }^{4}$

Teniendo en cuenta la excepcionalidad de la presentación de RCS y MCD en este rango etario, se describe la evolución del patrón respiratorio en una adolescente con insuficiencia cardíaca secundaria a MCD que, posteriormente, recibió trasplante cardíaco.

\section{Descripción del caso clínico}

Paciente femenino de 12 años de edad, con astenia, anorexia, ortopnea y edema de los miembros inferiores. Con diagnóstico de insuficiencia cardíaca congestiva (ICC) clase funcional IV, fue admitida en la Unidad de Cuidados Intensivos Pediátricos y presentó un ecocardiograma con fracción de acortamiento del $15 \%$ y requerimiento de inotrópicos endovenosos. La evaluación definió el diagnóstico de MCD de origen idiopático y fue incluida en la lista para trasplante cardíaco.

Durante su evolución, presentó dificultad para conciliar el sueño, despertares frecuentes y episodios de hipoxemia cíclicos, que fueron parcialmente resueltos por el uso de cánula de alto flujo de $1,5 \mathrm{l} / \mathrm{kg} / \mathrm{min}(40 \mathrm{l} / \mathrm{min})$ y oxígeno 
con fracción inspirada de oxígeno $\left(\mathrm{FiO}_{2}\right)$ del $30 \%$. Con sospecha de alteraciones respiratorias del sueño y ante la dificultad de realizar un estudio de polisomnografía dentro de la Unidad de Cuidados Intensivos, se realizó una poligrafía respiratoria (PR) nocturna con un dispositivo portátil nivel III (Academia Americana de Sueño, AAMS) Alice PDx Philips Respironics. Se incluyeron en el análisis cánula de presión nasal, bandas de esfuerzo torácica y abdominal, y oximetría con sensor dedal.

La grabación fue editada y analizada manualmente por un neumonólogo entrenado en el uso de estándares actuales de la AAMS. ${ }^{5}$ Debido al estado clínico de la paciente, no fue posible suspender la cánula de alto flujo para el registro. Este mostró un patrón típico de RCS con apneas centrales cíclicas que alternaban con un patrón respiratorio creciente-decreciente durante el $37 \%$ del tiempo de registro. Cada ciclo duraba una media de 54 segundos, y el duty ratio (DR) fue de 0,57 (Figura 1).

El índice de apneas centrales por hora/sueño (IAC) en eventos / hora fue de 6,6, elevado para la referencia en pediatría (valor normal: $<1 / \mathrm{h}$ ), ${ }^{6}$ con presencia de hipoxemia intermitente el 2,1 \% del tiempo total de registro (TTR) con saturación de oxígeno $\left(\mathrm{O}_{2}\right)<90 \%$ (mínima del $88 \%$ ), a pesar de la administración de $\mathrm{O}_{2}$ suplementario.
Evolucionó con shock cardiogénico refractario a la maximización de tratamientos habituales, por lo que recibió soporte circulatorio con oxigenación extracorpóreo (extracorporeal membrane oxygenation; ECMO, por sus siglas en inglés) y trasplante cardíaco 72 horas después.

La evolución posoperatoria fue adecuada con fracción de acortamiento del $55 \%$ a la semana. Dos semanas postrasplante, la PR sin oxígeno suplementario evidenció IAC/h de 1,4 sin hipoxemia. El monitoreo de la presión parcial de dióxido de carbono $\left(\mathrm{PCO}_{2}\right)$ al fin de la espiración mostró valores medios de normocapnia. En la semana 7 luego del trasplante, una nueva PR (Figura 2) mostró valores normales de IAC / h $(0,4)$ y saturación de $\mathrm{O}_{2}$ promedio del $98 \%$ (mínima del $94 \%$ ) sin evidencia de RCS (Tabla 1).

\section{DISCUSIÓN}

La RCS, caracterizada por un patrón ventilatorio periódico creciente-decreciente con apneas centrales y/o hipopneas centrales, representa una afección prevalente en adultos con insuficiencia cardíaca. ${ }^{1,2}$ Por el contrario, esta entidad es comunicada infrecuentemente en pediatría.

La MCD es un trastorno cardíaco grave en niños que origina ICC y falla orgánica múltiple, con una mortalidad del $20 \%$ al año y del $56 \%$

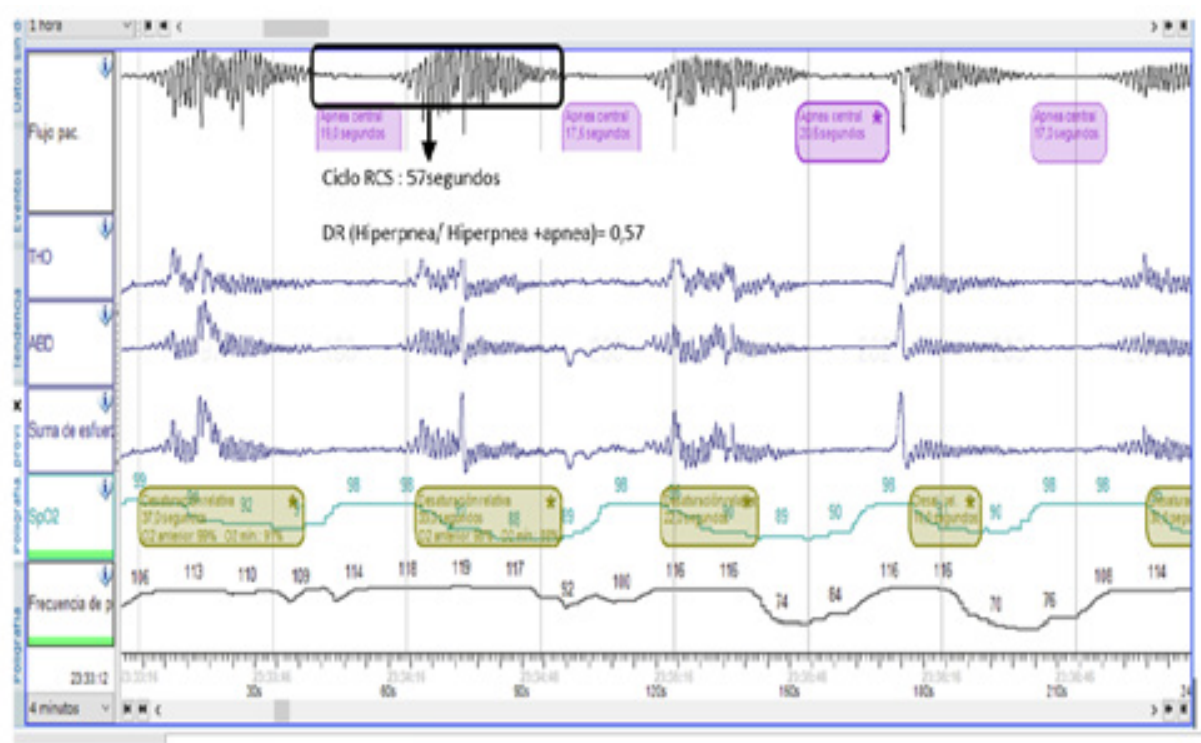

Nótese el patrón respiratorio característico y los eventos de apneas de mecanismo central (sin esfuerzo) con desaturaciones. 
a 4 años, que representa la principal indicación de trasplante cardíaco. ${ }^{7}$ Su incidencia estimada es de 0,57/100 000 personas por año. La forma idiopática constituye la variante más frecuente $(\mathrm{el} 70 \%)^{8}$

En el caso presentado, se observa RCS con ICC (clase funcional IV) secundario a MCD, que coincide con la descripción de Den Boer y cols., de 2016. ${ }^{9}$ En este estudio, se evaluaron 37 niños con ICC secundaria a MCD con PR, y se encontró el síndrome de apneas centrales en el $19 \%$ de la cohorte estudiada $(n=8)$. Solo tres pacientes, de 12, 15 y 16 años, presentaron RCS, y dos de ellos recibieron trasplante cardíaco. Estos autores hallaron menor frecuencia de RCS en relación con los adultos.

La fisiopatología de la RCS se explica por la teoría de la ganancia de bucle (loop gain; $L G$, por sus siglas en inglés), que involucra quimiorreceptores periféricos y centrales, receptores vagales intrapulmonares, centro respiratorio en el tronco encefálico y músculos respiratorios. En este modelo, el centro respiratorio y los pulmones mantienen una

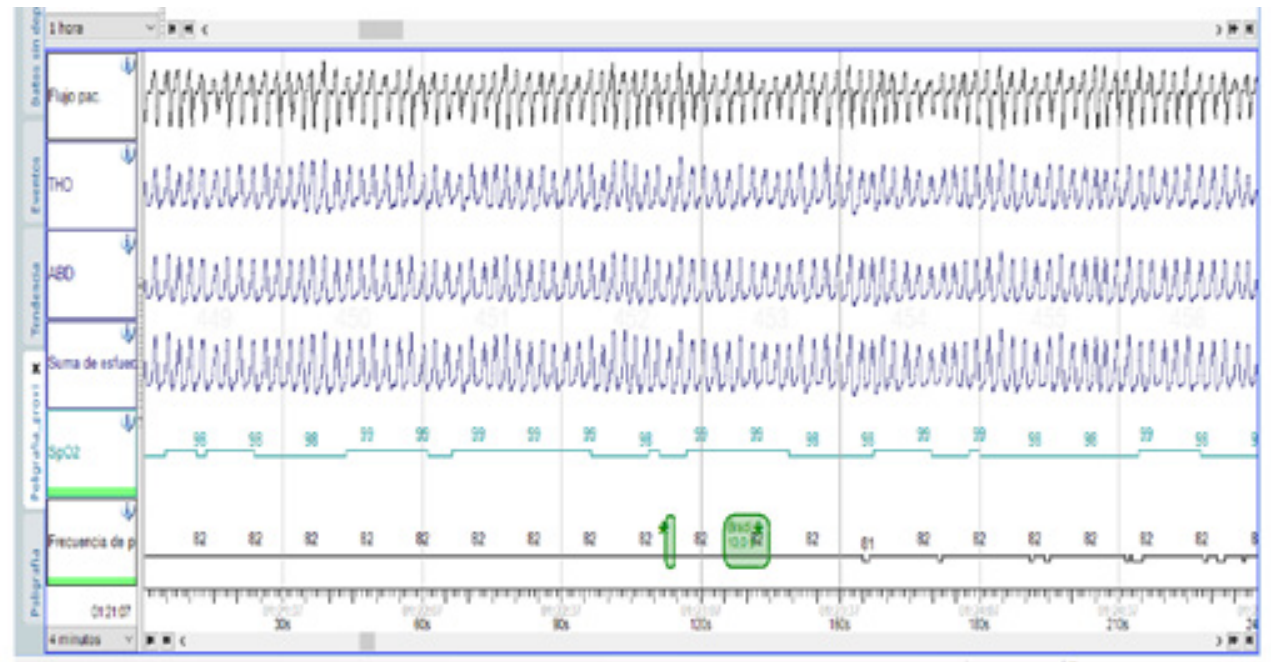

Nótese la resolución de las alteraciones respiratorias con una saturación media del 98 \% con $\mathrm{FiO}_{2}$ del $21 \%$.

TABLA 1. Indicadores poligráficos y ecocardiograma pre y pos trasplante cardíaco

\begin{tabular}{|c|c|c|c|}
\hline Indicadores & Pretrasplante & $\begin{array}{l}\text { Postrasplante } \\
\text { ( } 2^{\mathrm{a}} \text { semana) }\end{array}$ & $\begin{array}{l}\text { Postrasplante } \\
\text { ( } 7^{\mathrm{a}} \text { semana) }\end{array}$ \\
\hline TTR & 325 & 502 & 449 \\
\hline $\mathrm{IAC} / \mathrm{h}$ & 6,6 & 1,7 & 0,4 \\
\hline $\mathrm{IAHO} / \mathrm{h}$ & 1,8 & 0,1 & 0,7 \\
\hline $\mathrm{SaO}_{2}$ promedio & $98 \%\left(\mathrm{FiO}_{2} 30 \%\right)$ & $98 \%$ (AA) & $98 \%$ (AA) \\
\hline $\mathrm{SaO}_{2}$ mínima & $88 \%$ & $92 \%$ & $94 \%$ \\
\hline Respiración de Cheyne-Stokes & $120 \min (37 \%)$ & $23,74 \min (4,7 \%)$ & 0 \\
\hline Ciclo de RCS & 54 segundos & 47 segundos & - \\
\hline DR & 0,57 & 0,79 & - \\
\hline $\mathrm{ETCO}_{2}$ & -------- & $42 \mathrm{mmHg}$ & $38 \mathrm{mmHg}$ \\
\hline $\begin{array}{l}\text { Ecocardiograma } \\
\text { (\% fracción de acortamiento) }\end{array}$ & $15 \%$ & $53 \%$ & $55 \%$ \\
\hline
\end{tabular}

TTR: tiempo total de registro; IAC/h: índice de apneas centrales por hora de sueño; IAHO/h: índice de apneas/hipopneas obstructivas por hora de sueño; $\mathrm{SaO}_{2}$ : saturación de oxígeno; respiración de Cheyne-Stokes: expresada en minutos y porcentaje del tiempo total de registro; ciclo de RCS: longitud del ciclo desde el inicio de un evento de apnea hasta el siguiente o de pico a pico en patrones en forma de diamante expresada en segundos; DR: duty ratio; $\mathrm{ETCO}_{2}: \mathrm{CO}_{2}$ de fin de espiración o End Tidal $\mathrm{CO}_{2}$ en simultáneo con la poligrafía respiratoria nocturna; AA: aire ambiente equivalente a $\mathrm{FIO}_{2}$ del $21 \%$. 
relación recíproca de retroalimentación negativa para regular un parámetro clave, que es la $\mathrm{PCO}_{2}$ arterial. En este sistema de retroalimentación negativa, el aumento de la $\mathrm{PCO}_{2}$ es la señal que estimula los quimiorreceptores periféricos y centrales para aumentar la ventilación.

Luego de un tiempo de circulación, la sangre arterial hipocápnica es medida por los quimiorreceptores, lo que determina una reducción en el impulso ventilatorio, que, a su vez, elevará la $\mathrm{PCO}_{2}$ arterial. El aumento excesivo del impulso ventilatorio hasta descender la $\mathrm{PCO}_{2}$ debajo del umbral de apneas determina las apneas recurrentes cíclicamente. ${ }^{1}$ Este mecanismo fisiopatológico dependiente del valor de $\mathrm{CO}_{2}$ es responsable de la inestabilidad del sistema respiratorio.

Además, el retraso circulatorio modifica la respuesta a las variaciones de $\mathrm{PCO}_{2}$, como en nuestro caso, debido a ICC y fracción de eyección del ventrículo izquierdo baja, donde la información de la $\mathrm{PCO}_{2}$ llega con demora desde el pulmón hasta los quimiorreceptores, retrasa la respuesta y se manifiesta como RCS.

En los adultos, el $L G$ se obtiene por una compleja fórmula que, en la práctica clínica, es reemplazada por la medición en el trazado del $D R$, un subrogante cuya equivalencia se observa en la Tabla 2.10,11 Cuanto más se aleja de la unidad, hacia el cero, el sistema es más inestable, como se observó en el trazado de esta paciente, cuyo $D R$ de 0,57 segundos se prolongó a 0,79 luego del trasplante.

Esta compleja relación entre la RCS y la ICC, en la que las variaciones cíclicas en la frecuencia cardíaca, presión arterial, volumen respiratorio, presión parcial de $\mathrm{O}_{2} \mathrm{y} \mathrm{CO}_{2}$ conducen a la hiperactividad simpática, lo que empeora la contracción ventricular y la congestión pulmonar, propone desafíos terapéuticos con el objetivo de atenuar la respiración periódica y, en consecuencia, la actividad simpática. ${ }^{12}$ En los adultos, los más utilizados son la administración de oxígeno, la presión positiva continua en la vía aérea y la ventilación servo-adaptativa.
Sin embargo, Naughton ${ }^{13}$ ha postulado que la RCS que persiste a pesar de la "terapia óptima" de la ICC constituye en sí misma un mecanismo compensatorio en la ICC. Otros autores, como S. Javaheri y cols. ${ }^{14}$ rechazan esta hipótesis y consideran la RCS como un factor de mal pronóstico con mayor mortalidad. En la actualidad, ensayos clínicos aleatorizados con presión positiva continua en la vía aérea, ventilación servo-adaptativa, oxígeno suplementario intentan atenuar la hiperactividad simpática y revertir la RCS.

En este estudio, se observó una mejoría del disturbio respiratorio a las 2 semanas del trasplante cardíaco, que alcanzó la normalización a las 7 semanas. Este período fue menor que lo descrito por Suhail Al-Saleh y cols., a 3 meses en un paciente pediátrico. ${ }^{4}$ En los adultos, el tiempo de resolución de la RCS posterior al trasplante por diferentes etiologías ha sido comunicado a las 10 semanas. $^{15}$

La escasa evidencia de RCS en niños con ICC podría deberse a la infrecuente realización de estudios de sueño que permitirían diagnosticar esta afección. Finalmente, la realización de una evaluación nocturna en pacientes con ICC clínicamente estables, en quienes la identificación de RCS podría condicionar otras modalidades de tratamiento, requiere de mayores estudios para establecer su rol, teniendo en cuenta las controversias existentes en el abordaje de la RCS.

\section{REFERENCIAS}

1. Orr JE, Malhotra A, Sands SA. Pathogenesis of central and

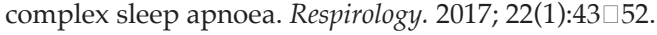

2. Berry RB, Brooks R, Gamaldo CE, Harding SM, et al. The AASM Manual for the Scoring of Sleep and Associated Events: Rules, Terminology and Technical Specifications. Version 2.0.1. Darien, Ill: American Academy of Sleep Medicine; 2013.

3. Javaheri S. Sleep disorders in systolic heart failure: a prospective study of 100 male patients. The final report. In J Cardiol. 2009; 106(1):21-8.

4. Al-Saleh S, Kantor PF, Narang I. Impact of Heart Transplantation on Cheyne-Stokes Respiration in a Child. Case Rep Pediatr. 2016; 2016:4698756.

5. Berry RB, Budhiraja R, Gottlieb DJ, Gozal D, et al. Rules for scoring respiratory events in sleep: update of the 2007

TABLA 2. Relación del loop gain y el duty ratio

\begin{tabular}{|c|c|c|c|c|c|c|c|c|c|c|c|}
\hline$L G$ & 1 & 1,001 & 1,001 & 1,037 & 1,088 & 1,175 & 1,311 & 1,523 & 1,852 & 2,379 & 3,263 \\
\hline$D R$ & 1 & 0,94 & 0,88 & 0,82 & 0,76 & 0,7 & 0,64 & 0,58 & 0,52 & 0,46 & 0,4 \\
\hline
\end{tabular}

Nótese la relación inversa: a mayor loop gain, menor duty ratio, en la que 1 es el valor de estabilidad para ambos.

LG: loop gain; DR: duty ratio. 
AASM Manual for the Scoring of Sleep and Associated Events. Deliberations of the Sleep Apnea Definitions Task Force of the American Academy of Sleep Medicine. J Clin Sleep Med. 2012; 8(5):597-619.

6. Uliel S, Tauman R, Greenfeld M, Sivan Y. Normal polysomnographic respiratory values in children and adolescents. Chest. 2004; 125(3):872-8.

7. Daubeney P, Nugent A, Chondros P, Carlin JB, et al. National Australian Childhood Cardiomyopathy Study. Clinical features and outcomes of childhood dilated cardiomyopathy: results from a national population-based study. Circulation. 2006; 114(24):2671-8.

8. Towbin JA, Lowe AM, ColanSD, Sleeper L, et al. Incidence, causes, and outcomes of dilated cardiomyopathy in children. JAMA. 2006; 296(15):1867-76.

9. Den Boer SL, Joosten KF, Van den Berg S, Backx AP, et al. Prospective evaluation of sleep apnea as manifestation of heart failure in children. Pediatr Cardiol. 2016;37(2):248 $\square 54$.

10. Sands SA, Edwards BA, KeeK, Turton A, et al. Loop gain as a means to predict a positive airway pressure suppression of Cheyne-Stokes respiration in patients with heart failure. Am J Respir Crit Care Med. 2011; 184(9):1067 $\square 75$.
11. Ortiz Naretto AE, Borsini E. Apnea central del sueño y Respiración periódica. Rev Amer Med Respiratoria. 2020; 20(2):150-61.

12. Terziyski K, Draganova A. Central Sleep Apnea with Cheyne-Stokes Breathing in Heart Failure-From Research to Clinical Practice and Beyond. In: Islam M (ed). Heart Failure: From Research to Clinical Practice. Advances in Experimental Medicine and Biology. Cham:Springer; 2018; 1067:327-51.

13. NaughtonMT.PRO:Persistent central sleep apnea/HunterCheyne-Stokes breathing, despite best guideline-based therapy of heart failure with reduced ejection fraction, is a compensatory mechanism and should not be suppressed. J Clin Sleep Med. 2018; 14(6):909 $\square 14$.

14. Javaheri S, Brown LK, Khayat R. CON: Persistent central sleep apnea/Hunter-Cheyne-Stokes breathing, despite best guideline-based therapy of heart failure with reduced ejection fraction, is not a compensatory mechanism and should be suppressed. J Clin Sleep Med. 2018; 14(6):915 $\square 21$.

15. Thalhofer SA, Kiwus U, Dorow P. Influence of orthotopic heart transplantation on breathing pattern disorders in patients with dilated cardiomyopathy. Sleep Breath. 2000; 4(3):121-6. 